\title{
Bevacizumab plus platinum-based chemotherapy in advanced non-squamous non-small-cell lung cancer: a randomized, open-label phase 2 study (CLEAR)
}

\author{
Hibiki Udagawa ${ }^{1}$, Eri Sugiyama ${ }^{1}$, Toshiyuki Harada ${ }^{2}$, Shinji Atagi $^{3}$, Ryo Koyama ${ }^{4}$, Satoshi Watanabe ${ }^{5}$, \\ Yukiko Nakamura ${ }^{6}$, Daijiro Harada ${ }^{7}$, Osamu Hataji ${ }^{8}$, Fumihiro Tanaka ${ }^{9}$, Hiroshi Kida ${ }^{10}$, \\ Miyako Satouchi ${ }^{11}$, Ken Maeno ${ }^{12}$, Akira Inoue ${ }^{13}$, Kiyotaka Yoh ${ }^{1}$, Yuki Yamane ${ }^{14}$, Yoshiko Urata ${ }^{11}$, \\ Hiroshige Yoshioka ${ }^{15}$, Takeharu Yamanaka ${ }^{16}$, Koichi Goto ${ }^{1}$
}

${ }^{1}$ Department of Thoracic Oncology, National Cancer Center Hospital East, 6-5-1 Kashiwanoha, Kashiwa, Chiba 277-8577, Japan; ${ }^{2}$ Department of Respiratory Medicine, Center for Respiratory Diseases, JCHO Hokkaido Hospital, 8-3-18 Nakanoshima 1-Jo, Sapporo, Hokkaido 062-8618, Japan; ${ }^{3}$ Department of Thoracic Oncology, Kinki-Chuo Chest Medical Center, 1180 Nagasonecho, Kita-ku, Sakai, Osaka 591-8555, Japan; ${ }^{4}$ Department of Respiratory Medicine, Juntendo University Faculty of Medicine, 2-1-1 Hongo, Bunkyo-ku, Tokyo 113-8421, Japan; ${ }^{5}$ Department of Respiratory Medicine and Infectious Diseases, Niigata University Graduate School of Medical and Dental Sciences, 754 Ichibancho, Asahimachidori, Chuoku, Niigata 951-8510, Japan; ${ }^{\circ}$ Department of Respiratory Medicine, Yokohama Municipal Citizen's Hospital, 1-1 Mitsuzawanishimachi, Kanagawaku, Yokohama, Kanagawa 221-0855, Japan; ${ }^{7}$ Department of Thoracic Oncology, Shikoku Cancer Center, 160 Minami Umemotomachi, Matsuyama, Ehime 791-0280, Japan; ${ }^{8}$ Respiratory Center, Matsusaka Municipal Hospital, 1550 Tonomachi, Matsusaka, Mie 515-8544, Japan; ${ }^{9}$ Second Department of Surgery, University of Occupational and Environmental Health, 1-1 Iseigaoka, Yahatanishi-ku, Kitakyushu, Fukuoka 807-8555, Japan; ${ }^{10}$ Department of Respiratory Medicine and Clinical Immunology, Osaka University Graduate School of Medicine, 2-2 Yamadaoka, Suita, Osaka 565-0871, Japan; ${ }^{11}$ Department of Thoracic Oncology, Hyogo Cancer Center, 13-70 Kitaojicho, Akashi, Hyogo 673-8558, Japan; ${ }^{12}$ Department of Respiratory Medicine, Allergy and Clinical Immunology, Nagoya City University Graduate School of Medical Science, 1 Kawasumi, Mizuho-cho, Mizuho-ku, Nagoya, Aichi 467-8602, Japan; ${ }^{13}$ Department of Palliative Medicine, Tohoku University School of Medicine, 2-1 Seiryo-machi, Aobaku, Sendai, Miyagi 980-8575, Japan; ${ }^{14}$ Department of Thoracic Oncology, Saitama Cancer Center, 780 Komuro, Inamachi, Kitaadachi-gun, Saitama 362-0806, Japan; ${ }^{15}$ Department of Thoracic Oncology, Kansai Medical University Hospital, 2-5-1 Shin-machi, Hirakata, Osaka 573-1010, Japan; ${ }^{16}$ Department of Biostatistics, Yokohama City University, 3-9 Fukuura, Kanazawa-ku, Yokohama, Kanagawa 236-0004, Japan

Contributions: (I) Conception and design: H Udagawa, E Sugiyama, A Inoue, K Yoh, Y Yamane, Y Urata, H Yoshioka, T Yamanaka, K Goto; (II) Administrative support: H Udagawa, E Sugiyama, K Goto; (III) Provision of study materials or patients: H Udagawa, E Sugiyama, T Harada, S Atagi, R Koyama, S Watanabe, Y Nakamura, D Harada, O Hataji, F Tanaka, H Kida, M Satouchi, K Maeno, A Inoue, K Yoh, Y Yamane, Y Urata, H Yoshioka, K Goto; (IV) Collection and assembly of data: H Udagawa, E Sugiyama, T Harada, S Atagi, R Koyama, S Watanabe, Y Nakamura, D Harada, O Hataji, F Tanaka, H Kida, M Satouchi, K Maeno, A Inoue, K Yoh, Y Yamane, Y Urata, H Yoshioka, K Goto; (V) Data analysis and interpretation: H Udagawa, E Sugiyama, T Yamanaka, K Goto; (VI) Manuscript writing: All authors; (VII) Final approval of manuscript: All authors. Correspondence to: Koichi Goto. Department of Thoracic Oncology, National Cancer Center Hospital East, 6-5-1 Kashiwanoha, Kashiwa, Chiba 277-8577, Japan. Email: kgoto@east.ncc.go.jp.

Background: Atezolizumab combined with bevacizumab plus platinum-based chemotherapy is a standard treatment for advanced non-squamous non-small-cell lung cancer (nsNSCLC). We aimed to determine the most effective platinum-based combination, such that future studies with atezolizumab can be conducted to further improve patient outcomes.

Methods: This phase 2 study enrolled treatment-naïve patients with advanced or recurrent nsNSCLC who were randomly assigned to either cisplatin $\left(75 \mathrm{mg} / \mathrm{m}^{2}\right)+$ pemetrexed $\left(500 \mathrm{mg} / \mathrm{m}^{2}\right)+$ bevacizumab $(15 \mathrm{mg} / \mathrm{kg})$ (CisPemBev) followed by maintenance PemBev $(\mathrm{N}=132)$ or carboplatin (area under the concentrationtime curve of $6 \mathrm{mg} / \mathrm{mL} / \mathrm{min})+$ paclitaxel $\left(200 \mathrm{mg} / \mathrm{m}^{2}\right)+$ bevacizumab $(15 \mathrm{mg} / \mathrm{kg})$ (CarPacBev) followed by maintenance Bev $(\mathrm{N}=67)$. The primary endpoint was progression-free survival (PFS, by central review). Secondary endpoints included overall survival (OS) and overall response rate (ORR). Adverse events (AEs) were evaluated for safety. This study was designed with the point estimate of the hazard ratio (HR) for PFS calculated based on an expected $\mathrm{HR}<0.830$ with a probability $\geq 80 \%$. 


\begin{abstract}
Results: The HR for PFS (CisPemBev/CarPacBev) was 0.825 [95\% confidence interval (CI), 0.600-1.134, median PFS, 7.6 vs. 7.0 months]. Because the observed point estimate of the HR for PFS was $<0.830$, the primary endpoint was met, and CisPem doublet therapy was deemed to be more effective than CarPac in terms of PFS. Median OS was 23.4 months for CisPemBev and 21.6 months for CarPacBev (HR 0.845; 95\% CI, 0.583-1.242). The ORR was 57\% for CisPemBev and 55\% for CarPacBev. Both CisPemBev and CarPacBev were well tolerated; grade $\geq 3 \mathrm{AEs}$ were reported in $67 \%$ and $82 \%$ of patients, respectively.

Conclusions: CisPem combined with Bev was more effective in improving PFS compared with CarPacBev in patients with advanced nsNSCLC. CisPemBev was also well tolerated by this patient population. A study to evaluate the efficacy of atezolizumab plus CisPemBev is warranted.
\end{abstract}

Trial Registration: University hospital Medical Information Network Clinical Trial Registry (ID: UMIN000013354).

Keywords: Bevacizumab; carcinoma; non-small cell lung; chemotherapy; phase II; survival

Submitted Mar 25, 2021. Accepted for publication May 31, 2021.

doi: $10.21037 /$ tlcr-21-240

View this article at: https://dx.doi.org/10.21037/tlcr-21-240

\section{Introduction}

Lung cancer is a leading cause of cancer deaths worldwide, and the survival rates are low even in developed countries (1). Non-small cell lung cancer (NSCLC) accounts for approximately $75-80 \%$ of lung cancer cases, and the majority of patients are at an advanced stage (IIIB/IV) when they are diagnosed $(2,3)$. In general, adenocarcinoma is the most common type of non-squamous (ns) NSCLC (4).

Bevacizumab is a humanized monoclonal antibody that targets vascular endothelial growth factor. Bevacizumab combined with platinum-doublet chemotherapy improves progression-free survival (PFS) and overall survival (OS) of patients with advanced nsNSCLC (5-8). Both carboplatin + paclitaxel + bevacizumab (CarPacBev) and carboplatin + pemetrexed + bevacizumab (CarPemBev) regimens are widely used in clinical practice (9). In a phase 3 study (PointBreak study), PFS was significantly improved with CarPemBev when compared with CarPacBev, but OS (the primary study endpoint) did not improve with the CarPemBev regimen compared with the CarPacBev regimen (10). Thus, CarPac is the most effective evidencebased regimen combined with Bev in advanced nsNSCLC.

However, cisplatin with pemetrexed (CisPem) is the most effective platinum-based chemotherapy for patients with advanced nsNSCLC and has shown better tolerability compared with other platinum-based regimens (11-14). Therefore, CisPem would appear to be a promising regimen for combination with Bev. In fact, in a singlearm phase 2 study investigating the efficacy and safety of
CisPemBev followed by PemBev in Japanese patients, PFS and OS were 12.0 and 31.0 months, respectively (15). The phase 3 AVAPERL clinical trial showed that CisPemBev induction therapy followed by PemBev maintenance therapy significantly prolonged PFS compared with Bev alone maintenance therapy (16). However, no study has been conducted to directly compare the efficacy and safety of CisPemBev and CarPacBev for advanced nsNSCLC.

Recently, anti-programmed cell death protein-1 (PD-1) or anti-programmed death-ligand 1 (PD-L1) plus platinumbased chemotherapy became a standard treatment for patients with advanced NSCLC $(17,18)$. In the phase 3 KEYNOTE-189 trial, the addition of pembrolizumab (an anti-PD-1 antibody) to CisPem or CarPem was shown to improve OS in patients with advanced NSCLC (17). Similarly, in the phase 3 IMpower150 study, the combination of atezolizumab (a humanized monoclonal antibody targeting PD-L1) and CarPacBev significantly improved OS in patients with advanced NSCLC (18). However, no study has evaluated the efficacy and safety of the combination of atezolizumab and CisPemBev.

This study aimed to select the most effective platinumbased regimen combined with bevacizumab with the intention of studying the combination of this platinumbased regimen with atezolizumab in the future. Thus, we compared the efficacy and safety of CisPemBev vs. CarPacBev in previously untreated advanced or recurrent nsNSCLC patients. We present the following article in accordance with the CONSORT reporting checklist 
(available at https://dx.doi.org/10.21037/tlcr-21-240).

\section{Methods}

\section{Study design and treatment}

This phase 2, randomized, multicenter, open-label clinical trial was conducted from May 2014 to April 2018. A list of participating centers is shown in Table S1. Eligible patients were centrally randomized to $\mathrm{CisPemBev}$ and $\mathrm{CarPacBev}$ at a ratio of 2:1 for induction therapy. Induction therapy consisted of four treatment cycles (one cycle: 21 days). Patients assigned to the CisPemBev group received cisplatin $\left(75 \mathrm{mg} / \mathrm{m}^{2}\right)$, pemetrexed $\left(500 \mathrm{mg} / \mathrm{m}^{2}\right)$, and bevacizumab $(15 \mathrm{mg} / \mathrm{kg}$ ) on Day 1 , and patients assigned to the CarPacBev group received carboplatin (area under the concentrationtime curve of $6 \mathrm{mg} / \mathrm{mL} / \mathrm{min})$, paclitaxel $\left(200 \mathrm{mg} / \mathrm{m}^{2}\right)$, and bevacizumab $(15 \mathrm{mg} / \mathrm{kg})$ on Day 1 .

After at least three cycles of induction therapy, patients were assessed for transitioning to maintenance therapy. Maintenance therapy consisted of bevacizumab $(15 \mathrm{mg} / \mathrm{kg})$ on Day 1 for patients in the CarPacBev group, whereas patients in the CisPemBev group received pemetrexed $\left(500 \mathrm{mg} / \mathrm{m}^{2}\right)$ and bevacizumab $(15 \mathrm{mg} / \mathrm{kg})$ on Day 1 . Maintenance therapy continued until disease progression or discontinuation due to the development of an adverse event (AE). Treatment after discontinuation due to an $\mathrm{AE}$ was not permitted until disease progression was reported. In this study, the electronic data capture (EDC) system was used to collect patient data. Investigators inputted the data manually with the support from the clinical support coordinator. Preparation, submission, modification and reviewing of case report forms were all carried out via the EDC system.

This trial was conducted in accordance with Good Clinical Practice Guidelines and conforms to provisions of the Declaration of Helsinki (as revised in Fortaleza, Brazil, October 2013). The protocol was approved by the Specified Non-profit Organization MINS Research Ethics Review Committee in Japan (IRB \#20000086), and informed consent was obtained from all the participants in the study. This study was registered at the University Hospital Medical Information Network Clinical Trial Registry (ID: UMIN000013354).

\section{Randomization}

Randomization was performed using an internet-based registration system once a physician confirmed patient eligibility. Randomization was stratified according to Eastern Cooperative Oncology Group performance status (ECOG PS) (0/1), tumor stage (IIIB/IV/recurrence after surgery), brain metastasis, and clinical center. Depending on the study site, participants were assigned to the intervention by a clinical research coordinator or a physician.

\section{Patients}

All patients had nsNSCLC, confirmed by histology or cytology, that was classified as stage IIIB/IV or had relapsed after surgery, had not received chemotherapy, and could not be administered radiotherapy. Inclusion criteria were as follows: aged 20 to 74 years; ECOG PS of $0-1$; measurable disease based on Response Evaluation Criteria in Solid Tumors (RECIST; version 1.1); epidermal growth factor receptor $(E G F R)$ mutation-negative (exon 19 deletion and exon $21 \mathrm{~L} 858 \mathrm{R}) ; A L K$ fusion gene-negative after genetic testing or unknown without testing; and adequate bone marrow, liver, and kidney function.

Exclusion criteria included the following: history or complication with hemoptysis $(>2.5 \mathrm{~mL})$ within the previous 3 months; treatment history of definitive or palliative radiotherapy to the chest; presence of tumor invasion to the hilar, heart, or large blood vessels; detectable tumor in the central bronchopulmonary segment; and symptomatic or non-symptomatic brain metastases, including patients with active steroid treatment to control the symptoms of brain metastases.

Prohibited concomitant medications and therapies included antitumor treatment other than protocol treatment, chemotherapy, hormone therapy, immunotherapy, antibody therapy, radiotherapy, thermotherapy, surgical treatment, beginning a new investigational drug, and other nonapproved drugs. Bisphosphonate formulations or an antireceptor activator of nuclear factor kappa-B ligand antibody, which are used for the symptomatic treatment of bone metastasis, were not prohibited.

\section{Endpoints and measurements}

The primary endpoint was PFS (centrally assessed). The secondary endpoints included investigator-assessed PFS and evaluation of OS, overall response rate (ORR), and safety profile.

The Union for International Cancer Control-TNM (2009 version) was used for tumor stage classification, the National Cancer Institute Common Terminology 
Criteria for Adverse Events version 4.0 (Japan Clinical Oncology Group version) was used to assess AEs collected from the start of therapy until 28 days after the final administration, and RECIST version 1.1 was used to judge tumor regression. Recorded AEs included those specific to bleeding and hemorrhaging (gastrointestinal, respiratory, urinary, reproductive organs, the central nervous system, and others), thrombosis and embolisms (arterial and venous side), perforations and fistulas (gastrointestinal, pulmonary, urinary, respiratory, urinary, and genital tract), hypertension, proteinuria, protracted wound healing, congestive heart failure, posterior reversible encephalopathy syndrome, and any other AEs that were classified as grade $\geq 3$.

PFS was assessed as the number of days from registration until disease progression or death, whichever came first. OS was assessed as the number of days from registration until death or the final survival confirmation day. ORR was assessed as the proportion of patients who achieved a complete or partial response.

\section{Statistical analysis}

The data cutoff was July 2017. For the present report, OS data were updated in April 2018. In first-line treatment for patients with NSCLC without gene mutations (e.g., EGFR mutation), achieving approximately a 2-month PFS median extension would lead to a clinically meaningful OS extension. When referring to the E4599 and PointBreak studies, if the median PFS was 5.6-6.0 months in the CarPacBev group, a 2-month extension in the CisPemBev group would result in a median PFS of approximately 8 months, with a hazard ratio (HR) of $0.7-0.75(5,10)$.

With a PFS HR of CisPemBev to CarPacBev set at 0.72, the point estimate of the HR was expected to be $<0.830$, as observed in the PointBreak study (10), with a probability $\geq 80 \%$. Thus, if the observed point estimate of the HR for PFS in this study was $<0.830$, the primary endpoint was met and CisPemBev would be deemed more effective than CarPacBev in terms of improving PFS. Based on these calculations, the necessary number of PFS events required was 170 . When considering the yearly dropout rate and randomization ratio $(2: 1)$, the target sample size was determined to be 210 patients.

The safety analysis set (SAF) included patients who received treatment at least once. The full analysis set (FAS) included patients who were included in the SAF, except for those diagnosed with an NSCLC other than nsNSCLC (as confirmed by histology or cytology) and those without an efficacy endpoint assessment after treatment started. Patients who died for any reason were included in the FAS.

The PFS and OS were analyzed using a log-rank test. HRs and $95 \%$ confidence intervals (CIs) were estimated using a Cox comparison hazard model. Survival curves were estimated using the Kaplan-Meier method. The 95\% CIs for ORR were analyzed using the Clopper-Pearson method. Forest plots of the HRs for PFS and OS were developed and stratified by patient characteristics, including the presence of brain metastases, which is an important prognostic factor that was also included as a factor for stratified randomization.

AEs were coded using the Medical Dictionary for Regulatory Activities (Japanese version 17.0) and are described by the System Organ Class and Preferred Terms. Events considered to be related to the study drug were counted separately as treatment-related AEs. Each laboratory test value course was collected as summary data. Analyses were carried out using SAS software version 9.2 (SAS Institute Inc., Cary, NC, USA).

\section{Results}

From May 2014 to May 2016, 199 patients were randomly assigned to receive CisPemBev ( $\mathrm{N}=132)$ or CarPacBev $(\mathrm{N}=67)$ (Figure 1). One patient from each group was found to be ineligible after randomization (one patient with alcohol hypersensitivity in the CarPacBev group and one patient with symptoms of brain metastases prior to treatment in the CisPemBev group). Both the SAF and FAS included the same number of patients: CisPemBev $(n=131)$ and CarPacBev $(\mathrm{n}=66)$. Two patients $(1.0 \%)$ were found to have $A L K$-positive tumors after registration but were still included in the analysis. Baseline patient and disease characteristics were similar between the two treatment groups (Table 1).

The median (95\% CI) follow-up duration was 20.6 months (19.7-22.8) and the median (range) number of cycles for protocol treatment (induction + maintenance therapies) was 7 [1-22] and 8 [1-51] cycles in the CarPacBev group and CisPemBev group, respectively. A total of 109 (83\%) patients in the CisPemBev group and 49 (74\%) patients in the $\mathrm{CarPacBev}$ group proceeded to maintenance treatment.

\section{Efficacy}

The target number of events (170 events) was achieved, with 171 events reported during the trial period. The HR for PFS 


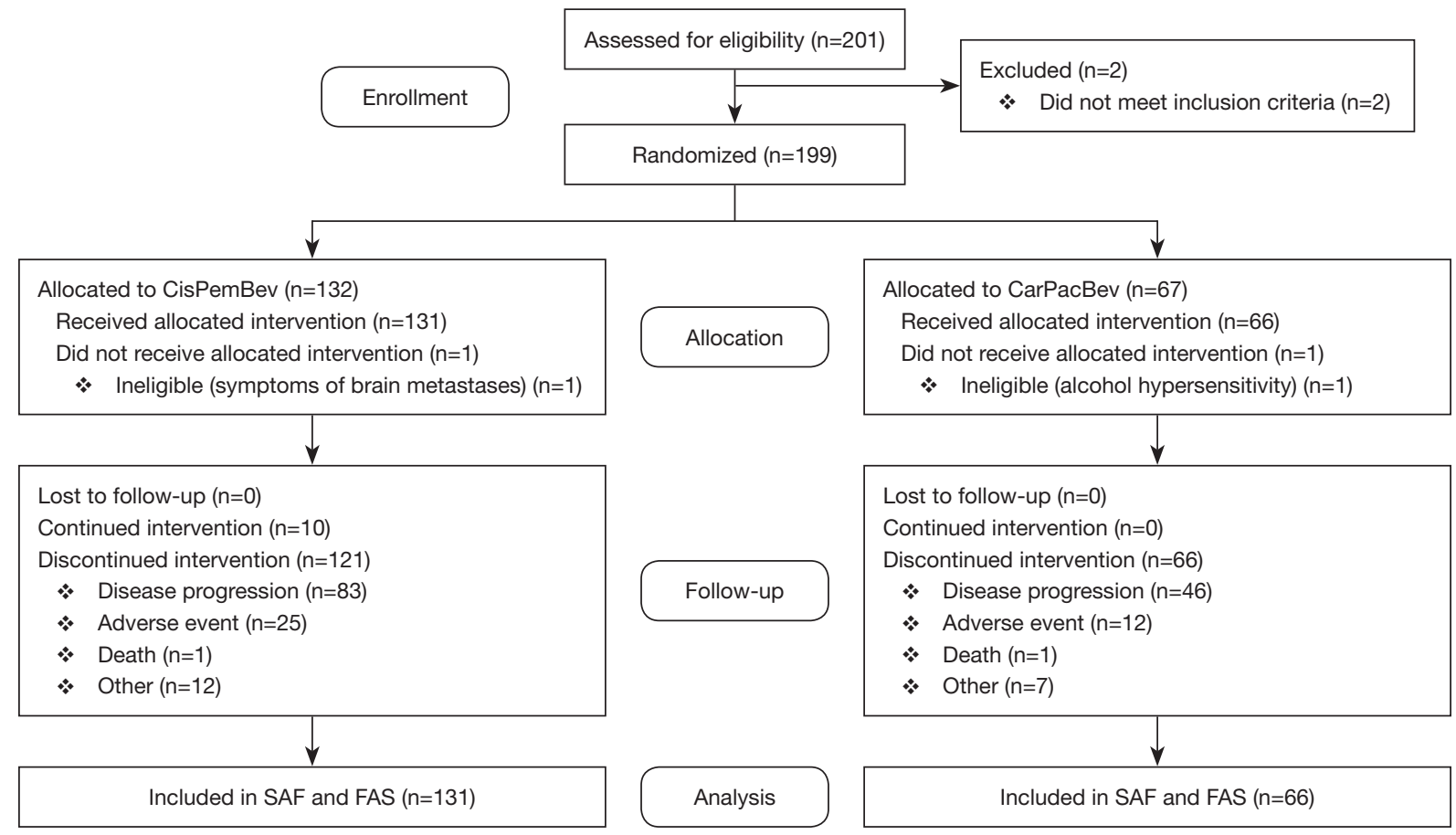

Figure 1 Participant flow. CisPemBev: cisplatin $75 \mathrm{mg} / \mathrm{m}^{2}$, pemetrexed $500 \mathrm{mg} / \mathrm{m}^{2}$, bevacizumab $15 \mathrm{mg} / \mathrm{kg}$. CarPacBev: carboplatin area under the concentration time curve of $6 \mathrm{mg} / \mathrm{mL} / \mathrm{min}$, paclitaxel $200 \mathrm{mg} / \mathrm{m}^{2}$, bevacizumab $15 \mathrm{mg} / \mathrm{kg}$.

by central review (CisPemBev/CarPacBev) was 0.825 (95\% CI, 0.600-1.134; median PFS, 7.6 vs. 7.0 months) (Figure 2A). The median PFS by investigator review was longer with CisPemBev than with CarPacBev (HR 0.634, 95\% CI, 0.464-0.867; median PFS, 7.4 vs. 6.8 months) (Figure 2B). The median OS was 23.4 months with CisPemBev and 21.6 months with CarPacBev (HR 0.845, 95\% CI, $0.583-1.242$ ) (Figure 2C). The ORR was $57 \%$ (95\% CI, 48-66\%) with CisPemBev and 55\% (95\% CI, 42-67\%) with CarPacBev.

Forest plots of the HRs for PFS and OS stratified by patient characteristics are shown in Figure 3A,B. The HRs for PFS and OS were lowest $(0.380,95 \%$ CI, 0.157-0.919 and $0.237,95 \%$ CI, 0.075-0.727, respectively) when stratified by tumors that were staged as recurrent.

Post-protocol treatments used in $\geq 5 \%$ of patients in the treatment groups are shown in Table S2. The majority of patients in both treatment groups received second-line post-protocol treatment (77\% in both the CisPemBev and CarPacBev groups). In addition, a PD-1 antibody (nivolumab or pembrolizumab) was administered to $49 \%$ of patients in the CisPemBev group, and $47 \%$ of patients in the CarPacBev group.
However, in the CisPemBev group, $28 \%$ of patients $(\mathrm{n}=37)$ received post-treatment before progressive disease (PD) was determined by central review, including an antiPD-1 antibody $(\mathrm{n}=10)$, pemetrexed regimen $(\mathrm{n}=8)$, taxane agent regimen $(n=17)$, or other $(n=2)$. In the $\mathrm{CarPacBev}$ group, $27 \%$ of patients $(n=18)$ received post-treatment before PD was determined by central review, including antiPD-1 antibody $(n=7)$, pemetrexed regimen $(n=8)$, taxane agent regimen $(n=2)$, or other $(n=1)$.

\section{Safety}

Grade $\geq 3$ AEs were reported in $67 \%$ of patients in the CisPemBev group and $82 \%$ of patients in the CarPacBev group. The most common grade $\geq 3$ AEs (CisPemBev/ CarPacBev) were neutrophil count decreased (24\%/64\%), white blood cell decreased (12\%/30\%), and hyponatremia (11\%/9\%) (Table 2). The most common Bev-related AEs (CisPemBev/CarPacBev) were hypertension (71\%/55\%), proteinuria $(50 \% / 55 \%)$, and epistaxis $(15 \% / 27 \%)$ (Table 3$)$.

Additional analysis indicated that there were no notable differences in the toxicity profiles according to patient age ( $<70$ and $\geq 70$ years; data not shown). 
Table 1 Baseline patient demographics and disease characteristics (both FAS and SAF)

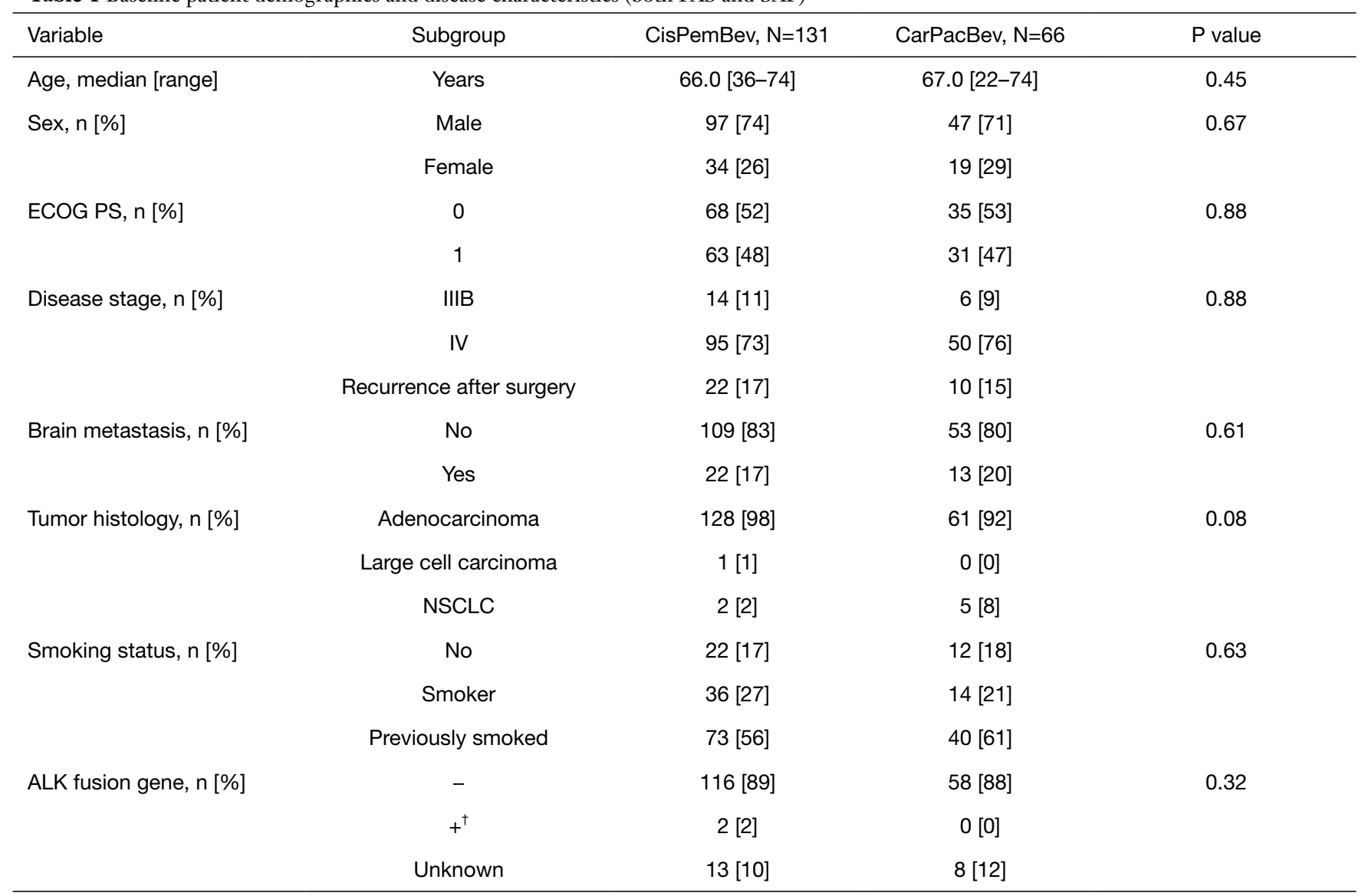

${ }^{\dagger}$, patients with ALK fusion gene-positive NSCLC were eligible if their ALK fusion gene status was unknown at study enrollment. ALK, anaplastic lymphoma kinase; CarPacBev, carboplatin + paclitaxel + bevacizumab; CisPemBev, cisplatin + pemetrexed + bevacizumab; ECOG PS, Eastern Cooperative Oncology Group performance status; FAS, full analysis set; NSCLC, non-small cell lung cancer; IQR, interquartile range; SAF, safety analysis set.

Treatment was discontinued due to an $\mathrm{AE}$ in $19 \%$ and $18 \%$ of patients in the CisPemBev and CarPacBev groups, respectively (Figure 1). Dose reduction due to an $\mathrm{AE}$ was required in $22 \%$ and $30 \%$ of patients in the CisPemBev and CarPacBev groups, respectively. Treatment-related deaths were reported in one patient (lung infection) in the CisPemBev group and one patient (enterocolitis) in the CarPacBev group. Fifteen patients in the CisPemBev group and one patient in the CarPacBev group developed treatment-related pneumonia. Of those, four patients in the CisPemBev group developed grade 3-4 treatment-related pneumonia.

\section{Discussion}

Recently, anti-PD-1 or anti-PD-L1 plus platinum-doublet chemotherapy showed durable clinical benefits and became a standard treatment for patients with advanced NSCLC $(17,18)$. The addition of atezolizumab to bevacizumab plus platinum-doublet chemotherapy significantly improved PFS and OS in patients with metastatic nsNSCLC (18). Thus, it is important to investigate the most effective bevacizumabcontaining platinum-based regimen with the intention of studying the combination of this platinum-based regimen with atezolizumab in the future. In this study, we compared the efficacy and safety of CisPemBev, the most promising platinum-based chemotherapy regimen combined with bevacizumab, with that of CarPacBev, the most effective evidence-based regimen combined with bevacizumab, to select the most effective bevacizumab-containing platinumbased regimen. A total of 199 treatment-naïve patients with advanced and recurrent nsNSCLC were enrolled, and the 
A
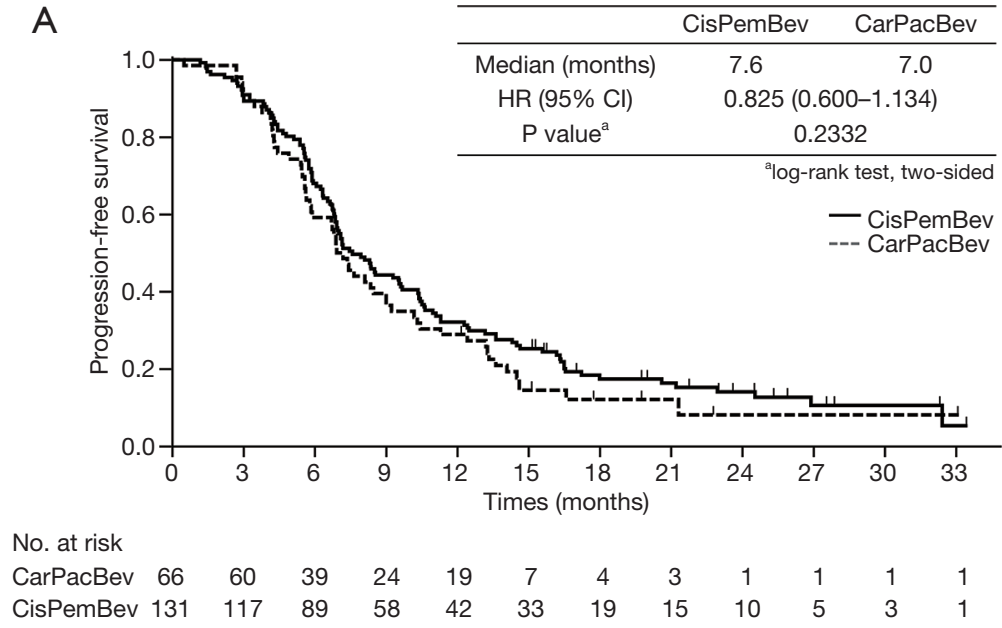

\section{B}
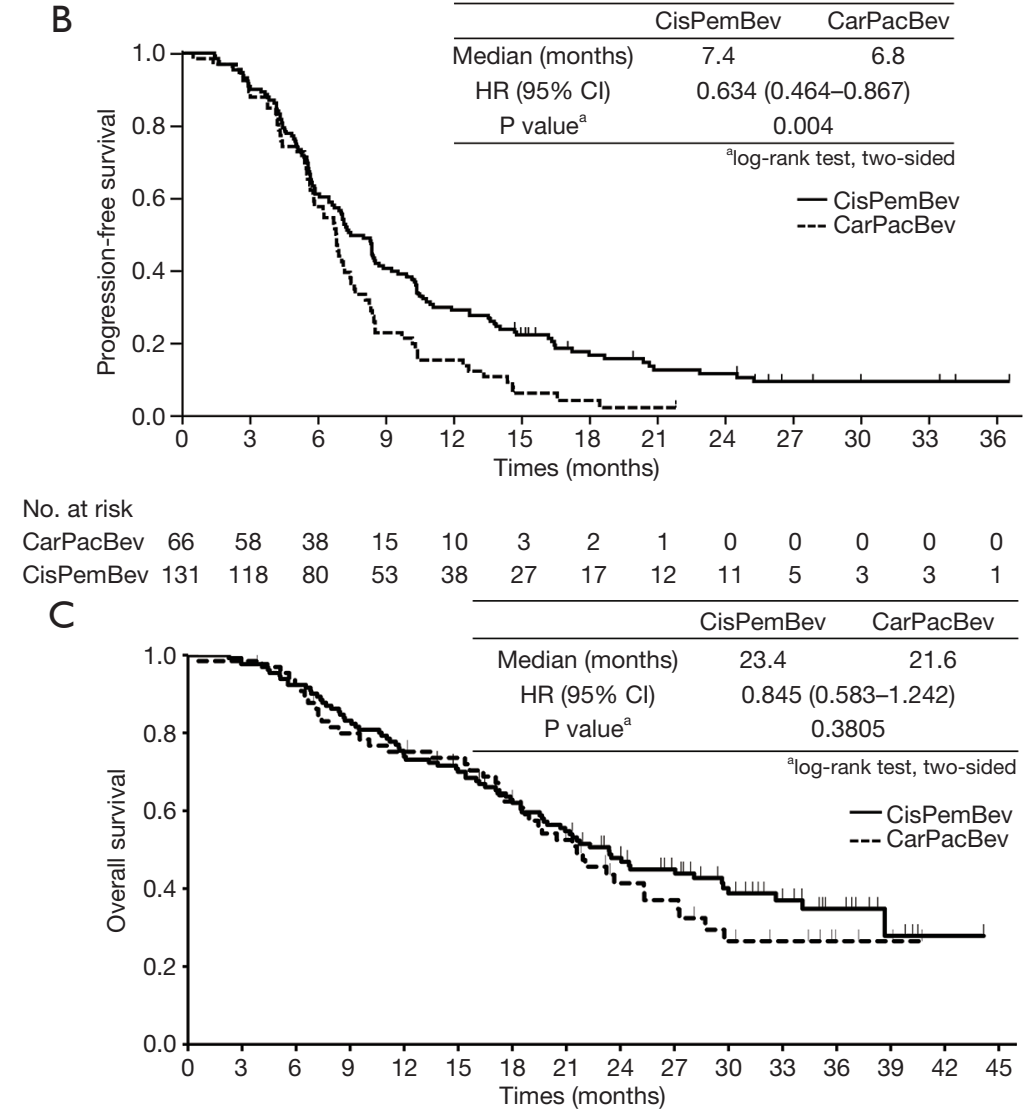

No. at risk

$\begin{array}{lllllllllllllllll}\text { CarPacBev } & 66 & 65 & 60 & 51 & 48 & 46 & 39 & 31 & 19 & 16 & 9 & 7 & 3 & 2 & 0 & 0\end{array}$

Figure 2 Kaplan-Meier curves of PFS by central review (A) and by investigator assessment (B); Kaplan-Meier curves of overall survival (C) (full analysis set). CarPacBev, carboplatin + paclitaxel + bevacizumab; CisPemBev, cisplatin + pemetrexed + bevacizumab; CI, confidence interval; HR, hazard ratio; PFS, progression-free survival. 


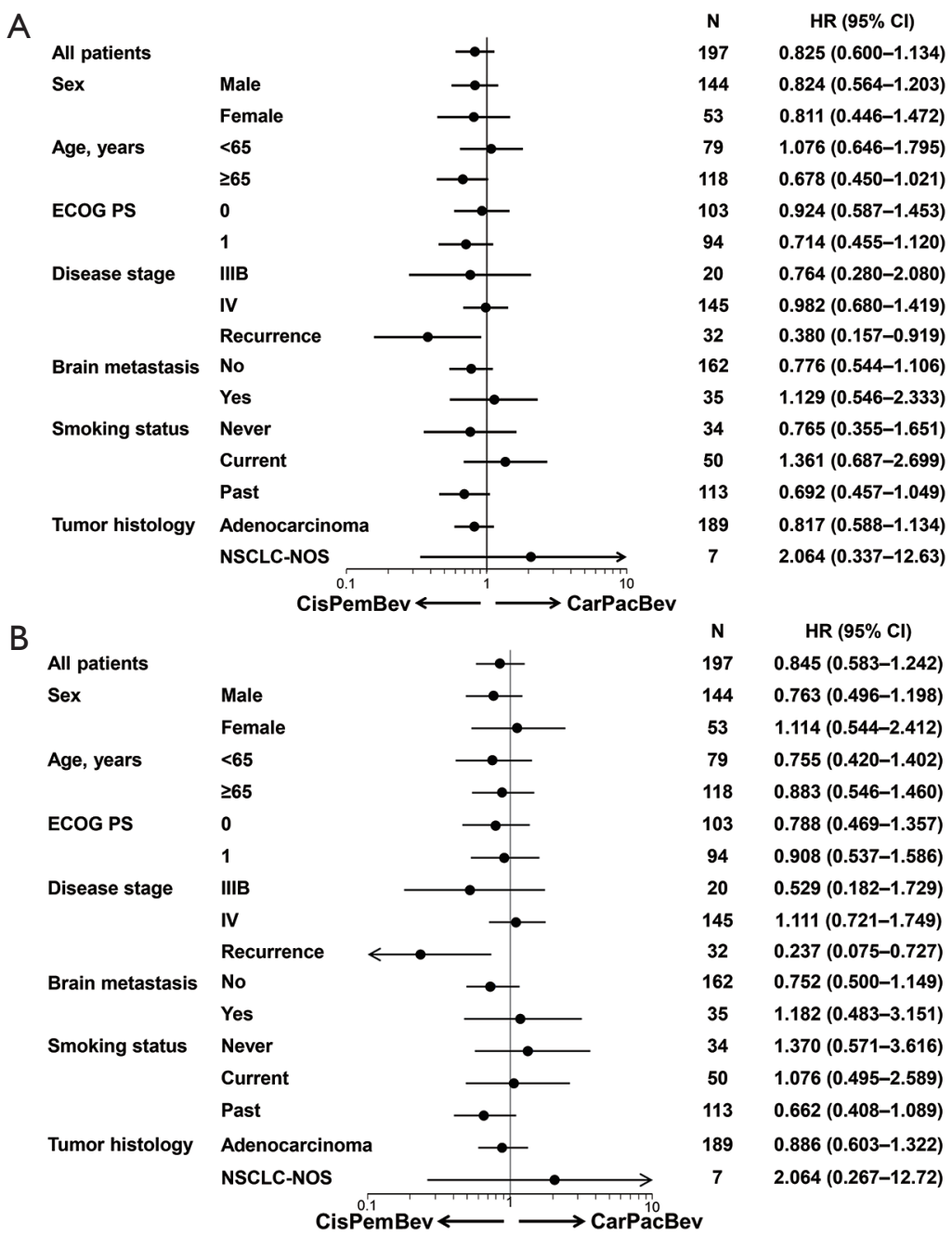

Figure 3 Forest plots of progression-free survival (A) and overall survival (B) by central review and stratified by patient characteristics. CarPacBev, carboplatin + paclitaxel + bevacizumab; CI, confidence interval; CisPemBev, cisplatin + pemetrexed + bevacizumab; ECOG PS, Eastern Cooperative Oncology Group performance status; HR, hazard ratio; NSCLC-NOS, non-small cell lung cancer not otherwise specified.

target number of events to have sufficient statistical power was achieved, with 171 events reported during the trial period. The point estimate of the HR for PFS was calculated based on an expected HR of $<0.830$ with a probability of $\geq 80 \%$. We concluded that the primary endpoint was met, because the HR was 0.825 , suggesting a greater efficacy of CisPemBev over CarPacBev in terms of PFS.

The PFS in the CisPemBev group of the present study was longer than that in the CarPemBev group in the PointBreak study (7.6 vs. 6.0 months) (10). In addition, the PFS of 7.6 months in the CisPemBev group in this study was very close to 8.0 months, which we expected.
CisPemBev treatment in this study also achieved a longer PFS than CisPem or CarPem induction treatment followed by Pem maintenance therapy in other studies $(19,20)$. In the present study, the PFS with CarPacBev was 7.0 months, which was longer than that reported in the E4599 study (5) and the PointBreak study (10), and was comparable with the expected PFS of 5.6-6.0 months. This is a possible reason for the small gap in PFS shown between CisPemBev (7.6 months) and CarPacBev (7.0 months) in the present study.

The OS of patients in both the CisPemBev and CarPacBev groups in this study was longer than the OS reported in the 
Table 2 Incidence of grade 3-5 adverse events (occurring in $>5 \%$ of patients)

\begin{tabular}{|c|c|c|}
\hline Adverse event & CisPemBev, N=131, n [\%] & CarPacBev, N=66, n [\%] \\
\hline Neutrophil count decreased & 32 [24] & $42[64]$ \\
\hline Anemia & $9[7]$ & $4[6]$ \\
\hline Platelet count decreased & $8[6]$ & 1 [2] \\
\hline White blood cell decreased & 16 [12] & $20[30]$ \\
\hline \multicolumn{3}{|l|}{ Non-hematological toxicities } \\
\hline Hyponatremia & $14[11]$ & $6[9]$ \\
\hline Anorexia & $9[7]$ & $3[5]$ \\
\hline
\end{tabular}

CarPacBev, carboplatin + paclitaxel + bevacizumab; CisPemBev, cisplatin + pemetrexed + bevacizumab.

Table 3 Summary of bevacizumab-related adverse events

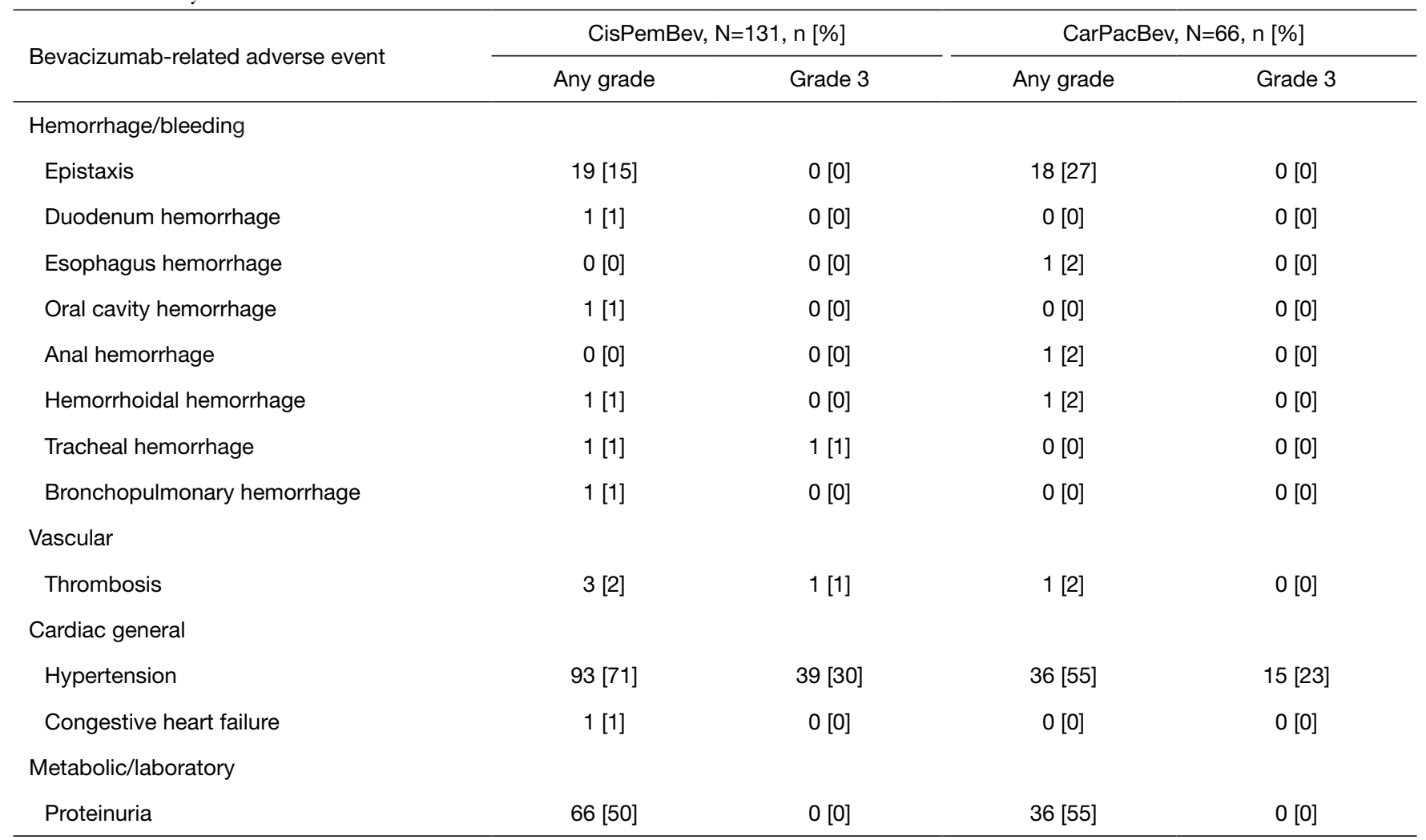

There were no grade 4 or 5 bevacizumab-related events. Common Terminology Criteria for Adverse Events v3.0. CarPacBev, carboplatin + paclitaxel + bevacizumab; CisPemBev, cisplatin + pemetrexed + bevacizumab.

PointBreak study (10). In this study, $77 \%$ of patients received some form of post-protocol treatment, compared with $<60 \%$ in the PointBreak study (10). Specifically, approximately half of the patients were administered PD-1 antibody treatments (nivolumab or pembrolizumab), which is a possible reason for an OS extension. Of note, this study was not influenced by the effect of EGFR tyrosine kinase inhibitors on PFS and OS extension because we excluded patients with EGFR gene 
mutations, unlike the JO19907 or BEYOND studies $(7,21)$.

When comparing the safety profiles of CisPemBev and CarPacBev in this study, the most common grade $\geq 3 \mathrm{AEs}$ in both arms were neutrophil count decreased, hyponatremia, and hypertension. However, the incidence rates were different; in particular, there were more cases of neutrophil count decreased in the CarPacBev group. Neutrophil count decreased is a frequent $\mathrm{AE}$ associated with CarPacBev therapy as has been previously demonstrated $(5,10,19)$. The most common Bev-related AEs were hypertension, proteinuria, and epistaxis; however, the incidence of epistaxis was more frequent in the $\mathrm{CarPacBev}$ group. This study also included 35 patients with brain metastasis (CisPemBev group, $\mathrm{n}=22$; CarPacBev group, $\mathrm{n}=13$ ); however, there were no reports of cerebral hemorrhage events.

It should be noted that although CisPem is an effective platinum-based chemotherapy for patients with advanced nsNSCLC, and has generally good tolerability compared with other platinum-based regimens, the possibility of Cis toxicity is a specific concern in elderly patients. Nonetheless, an analysis of two phase 3 trials of Cis doublet therapies in patients with nsNSCLC and good ECOG PS [0-1] concluded that CisPem was a viable treatment option in elderly patients (22). In that analysis, when patients were evaluated by age (either $<65$ and $\geq 65$ years, or $<70$ and $\geq 70$ years), toxicities were found to be manageable and similar between the younger and older age groups. In our study, an analysis by patient age found that there were no differences in CisPem toxicity in patients $<70$ and $\geq 70$ years of age. As a result, we can also conclude that CisPem is a viable treatment option with a manageable toxicity profile for elderly patients with nsNSCLC and good PS.

The limitations of the present study include those inherent to the open-label phase 2 study design. This study was also limited by insufficient statistical power to identify a significant difference in OS between the two treatment groups. The reason the sample size $(\mathrm{N}=199)$ was smaller than planned $(\mathrm{N}=210)$ was because the authors judged there would only be a few dropouts. However, the target number of events was achieved, giving the study sufficient statistical power for PFS evaluation. Finally, details of treatment-related grade 1-2 AEs were collected only for bevacizumab, and there were no corresponding data for the chemotherapeutic agents. Thus, we were unable to determine any differences in the frequency or type of grade 1-2 AEs between CisPem and CarPac in this study.

In conclusion, the PFS was improved in the CisPemBev group compared with the $\mathrm{CarPacBev}$ group. The safety profiles were different between both treatment regimens, and CisPemBev was well tolerated. CisPemBev is the most promising regimen to combine with atezolizumab for advanced nsNSCLC. A study to evaluate the addition of atezolizumab to CisPemBev is warranted.

\section{Acknowledgments}

The authors thank all patients, their family members, the investigators, and coordinators involved in this study. We also wish to thank Hikari Chiba and James Graham, $\mathrm{PhD}$, of Edanz Pharma for providing medical writing assistance, funded by Chugai Pharmaceutical Co., Ltd. This study has been presented in part at the World Conference on Lung Cancer 2017 and 2018, the Japan Lung Cancer Society 2017 and 2018, and the European Society for Medical Oncology 2018 Congress.

Funding: This work was supported by Chugai Pharmaceutical Co., Ltd. The sponsor was not involved in the study design, data collection, analysis, or interpretation, or manuscript development.

\section{Footnote}

Reporting Checklist: The authors have completed the CONSORT reporting checklist. Available at https://dx.doi. org/10.21037/tlcr-21-240

Trial Protocol: Available at https://dx.doi.org/10.21037/tlcr$21-240$

Data Sharing Statement: Available at https://dx.doi. org/10.21037/tlcr-21-240

Conflicts of Interest: All authors have completed the ICMJE uniform disclosure form (available at https://dx.doi. org/10.21037/tlcr-21-240). SW, FT, MS, HY, TY and KG have received honoraria from Chugai Pharmaceutical Co., Ltd. SA and KG have received research funds from Chugai Pharmaceutical Co., Ltd. FT, MS and TY have received scholarship endowments from Chugai Pharmaceutical Co., Ltd. The authors have no other conflicts of interest to declare.

Ethical Statement: The authors are accountable for all aspects of the work in ensuring that questions related to the accuracy or integrity of any part of the work are appropriately investigated and resolved. This trial was 
conducted in accordance with Good Clinical Practice Guidelines and conforms to provisions of the Declaration of Helsinki (as revised in Fortaleza, Brazil, October 2013). The protocol was approved by the Specified Non-profit Organization MINS Research Ethics Review Committee in Japan (IRB \#20000086), and informed consent was obtained from all the participants in the study.

Open Access Statement: This is an Open Access article distributed in accordance with the Creative Commons Attribution-NonCommercial-NoDerivs 4.0 International License (CC BY-NC-ND 4.0), which permits the noncommercial replication and distribution of the article with the strict proviso that no changes or edits are made and the original work is properly cited (including links to both the formal publication through the relevant DOI and the license). See: https://creativecommons.org/licenses/by-nc-nd/4.0/.

\section{References}

1. Torre LA, Siegel RL, Ward EM, et al. Global Cancer Incidence and Mortality Rates and Trends--An Update. Cancer Epidemiol Biomarkers Prev 2016;25:16-27.

2. Travis WD, Brambilla E, Muller-Hemerlink WK, et al. Pathology and Genetics of Tumours of the Lung, Pleura, Thymus and Heart. Oxford University Press, Oxford, 2004.

3. Molina JR, Yang P, Cassivi SD, et al. Non-small cell lung cancer: epidemiology, risk factors, treatment, and survivorship. Mayo Clin Proc 2008;83:584-94.

4. Cheng TY, Cramb SM, Baade PD, et al. The International Epidemiology of Lung Cancer: Latest Trends, Disparities, and Tumor Characteristics. J Thorac Oncol 2016;11:1653-71.

5. Sandler A, Gray R, Perry MC, et al. Paclitaxel-carboplatin alone or with bevacizumab for non-small-cell lung cancer. N Engl J Med 2006;355:2542-50.

6. Reck M, von Pawel J, Zatloukal P, et al. Phase III trial of cisplatin plus gemcitabine with either placebo or bevacizumab as first-line therapy for nonsquamous non-small-cell lung cancer: AVAil. J Clin Oncol 2009;27:1227-34.

7. Niho S, Kunitoh H, Nokihara H, et al. Randomized phase II study of first-line carboplatin-paclitaxel with or without bevacizumab in Japanese patients with advanced non-squamous non-small-cell lung cancer. Lung Cancer 2012;76:362-7.

8. Soria JC, Mauguen A, Reck M, et al. Systematic review and meta-analysis of randomised, phase II/III trials adding bevacizumab to platinum-based chemotherapy as first-line treatment in patients with advanced non-small-cell lung cancer. Ann Oncol 2013;24:20-30.

9. Abernethy AP, Arunachalam A, Burke T, et al. Real-world first-line treatment and overall survival in non-small cell lung cancer without known EGFR mutations or ALK rearrangements in US community oncology setting. PLoS One 2017;12:e0178420.

10. Patel JD, Socinski MA, Garon EB, et al. PointBreak: a randomized phase III study of pemetrexed plus carboplatin and bevacizumab followed by maintenance pemetrexed and bevacizumab versus paclitaxel plus carboplatin and bevacizumab followed by maintenance bevacizumab in patients with stage IIIB or IV nonsquamous non-small-cell lung cancer. J Clin Oncol 2013;31:4349-57.

11. Scagliotti GV, Parikh P, von Pawel J, et al. Phase III study comparing cisplatin plus gemcitabine with cisplatin plus pemetrexed in chemotherapy-naive patients with advanced-stage non-small-cell lung cancer. J Clin Oncol 2008;26:3543-51.

12. Ciuleanu T, Brodowicz T, Zielinski C, et al. Maintenance pemetrexed plus best supportive care versus placebo plus best supportive care for non-small-cell lung cancer: a randomised, double-blind, phase 3 study. Lancet 2009;374:1432-40.

13. Hanna N, Shepherd FA, Fossella FV, et al. Randomized phase III trial of pemetrexed versus docetaxel in patients with non-small-cell lung cancer previously treated with chemotherapy. J Clin Oncol 2004;22:1589-97.

14. Paz-Ares L, de Marinis F, Dediu M, et al. Maintenance therapy with pemetrexed plus best supportive care versus placebo plus best supportive care after induction therapy with pemetrexed plus cisplatin for advanced non-squamous non-small-cell lung cancer (PARAMOUNT): a doubleblind, phase 3, randomised controlled trial. Lancet Oncol 2012;13:247-55.

15. Fukushima T, Wakatsuki Y, Kobayashi T, et al. Phase II study of cisplatin/pemetrexed combined with bevacizumab followed by pemetrexed/bevacizumab maintenance therapy in patients with EGFR-wild advanced non-squamous nonsmall cell lung cancer. Cancer Chemother Pharmacol 2018;81:1043-50.

16. Barlesi F, Scherpereel A, Rittmeyer A, et al. Randomized phase III trial of maintenance bevacizumab with or without pemetrexed after first-line induction with bevacizumab, cisplatin, and pemetrexed in advanced nonsquamous nonsmall-cell lung cancer: AVAPERL (MO22089). J Clin 
Oncol 2013;31:3004-11.

17. Gandhi L, Rodríguez-Abreu D, Gadgeel S, et al. Pembrolizumab plus Chemotherapy in Metastatic NonSmall-Cell Lung Cancer. N Engl J Med 2018;378:2078-92.

18. Socinski MA, Jotte RM, Cappuzzo F, et al. Atezolizumab for First-Line Treatment of Metastatic Nonsquamous NSCLC. N Engl J Med 2018;378:2288-301.

19. Galetta D, Cinieri S, Pisconti S, et al. Cisplatin/ Pemetrexed Followed by Maintenance Pemetrexed Versus Carboplatin/Paclitaxel/Bevacizumab Followed by Maintenance Bevacizumab in Advanced Nonsquamous Lung Cancer: The GOIM (Gruppo Oncologico Italia Meridionale) ERACLE Phase III Randomized Trial. Clin Lung Cancer 2015;16:262-73.

20. Zinner RG, Obasaju CK, Spigel DR, et al. PRONOUNCE: randomized, open-label, phase III

Cite this article as: Udagawa $\mathrm{H}$, Sugiyama E, Harada T, Atagi S, Koyama R, Watanabe S, Nakamura Y, Harada D, Hataji O, Tanaka F, Kida H, Satouchi M, Maeno K, Inoue A, Yoh K, Yamane Y, Urata Y, Yoshioka H, Yamanaka T, Goto K. Bevacizumab plus platinum-based chemotherapy in advanced non-squamous non-small-cell lung cancer: a randomized, open-label phase 2 study (CLEAR). Transl Lung Cancer Res 2021;10(7):3059-3070. doi: 10.21037/tlcr-21-240 study of first-line pemetrexed + carboplatin followed by maintenance pemetrexed versus paclitaxel + carboplatin + bevacizumab followed by maintenance bevacizumab in patients ith advanced nonsquamous non-small-cell lung cancer. J Thorac Oncol 2015;10:134-42.

21. Zhou C, Wu YL, Chen G, et al. BEYOND: A Randomized, Double-Blind, Placebo-Controlled, Multicenter, Phase III Study of First-Line Carboplatin/ Paclitaxel Plus Bevacizumab or Placebo in Chinese Patients With Advanced or Recurrent Nonsquamous NonSmall-Cell Lung Cancer. J Clin Oncol 2015;33:2197-204.

22. Gridelli C, Brodowicz T, Langer CJ, et al. Pemetrexed therapy in elderly patients with good performance status: analysis of two phase III trials of patients with nonsquamous non-small-cell lung cancer. Clin Lung Cancer 2012;13:340-6. 
Supplementary

Table S1 List of participating centers

Department name, center name

Location (city, country)

Department of Thoracic Oncology, National Cancer Center Hospital East

Chiba, Japan

Department of Respiratory Medicine, Center for Respiratory Diseases, JCHO Hokkaido Hospital

Sapporo, Japan

Department of Thoracic Oncology, Kinki-Chuo Chest Medical Center

Sakai, Japan

Department of Respiratory Medicine, Juntendo University Faculty of Medicine

Department of Respiratory Medicine and Infectious Diseases, Niigata University Graduate School of Medical and Dental Sciences

Department of Respiratory Medicine, Yokohama Municipal Citizen's Hospital

Tokyo, Japan

Niigata, Japan

Department of Thoracic Oncology, Shikoku Cancer Center

Respiratory Center, Matsusaka Municipal Hospital

Second Department of Surgery, University of Occupational and Environmental Health

Department of Respiratory Medicine, Allergy and Clinical Immunology, Nagoya City University Graduate

School of Medical Science

Department of Respiratory Medicine and Clinical Immunology, Osaka University Graduate School of Medicine

Department of Thoracic Oncology, Hyogo Cancer Center

Department of Respiratory Medicine, Osaka Police Hospital

Department of Thoracic Oncology, National Hospital Organization Toneyama National Hospital

Department of Respiratory Medicine, Kurashiki Central Hospital

Respiratory Center, Asahikawa Medical University

Department of Thoracic Oncology, Kanagawa Cancer Center

Division of Medical Oncology, Department of Medicine, Showa University School of Medicine

Department of Respiratory Medicine, National Hospital Organization Hirosaki Hospital

Department of Respiratory Medicine, Kanagawa Cardiovascular and Respiratory Center

Center for Pulmonary Diseases, National Hospital Organization Tokyo National Hospital

Division of Respirology, Chiba Cancer Center

Department of Pulmonary Medicine, Graduate School of Medical and Dental Sciences, Kagoshima University

Department of Pulmonary Medicine, Sendai Kousei Hospital

Department of Respiratory Medicine, Nagasaki Prefecture Shimabara Hospital

Department of Respiratory Medicine, Miyagi Cancer Center

Department of Pulmonary Medicine, National Hospital Organization Matsue Medical Center

Department of Respiratory Medicine, National Hospital Organization Kyushu Medical Center

Department of Pulmonary Medicine, Fukushima Medical University Hospital

Department of Respiratory Medicine, Kobe Red Cross Hospital

Yokohama, Japan

Matsuyama, Japan

Matsusaka, Japan

Kitakyushu, Japan

Nagoya, Japan

Osaka, Japan

Akashi, Japan

Osaka, Japan

Toyonaka, Japan

Kurashiki, Japan

Asahikawa, Japan

Yokohama, Japan

Tokyo, Japan

Hirosaki, Japan

Yokohama, Japan

Tokyo, Japan

Chiba, Japan

Kagoshima, Japan

Sendai, Japan

Shimabara, Japan

Natori, Japan

Matsue, Japan

Fukuoka, Japan

Fukushima, Japan

Kobe, Japan

Internal Medicine I, Hokkaido University Hospital

Sapporo, Japan

Division of Pulmonary Medicine, Jichi Medical University Hospital

Shimotsuke, Japan

Tokyo, Japan

Department of Respiratory Medicine and Allergy, Tosei, Tosei General Hospital

Kanazawa, Japan

Table S1 (continued)

(c) Translational Lung Cancer Research. All rights reserved. 
Table S1 (continued)

\begin{tabular}{|c|c|}
\hline Department name, center name & Location (city, country) \\
\hline Division of Respiratory and Infectious Diseases, St Marianna University School of Medicine & Kawasaki, Japan \\
\hline Department of Thoracic Oncology, Aichi Cancer Center Hospital & Nagoya, Japan \\
\hline Department of Respiratory Medicine, Japanese Red Cross Nagoya Daiichi Hospital & Nagoya, Japan \\
\hline Department of Respiratory Medicine, Matsunami General Hospital & Gifu, Japan \\
\hline Department of Medical Oncology, National Hospital Organization Yamaguchi-Ube Medical Center & Ube, Japan \\
\hline Respiratory Medicine Section, Fukujuji Hospital, Japan Anti-Tuberculosis Association & Kiyose, Japan \\
\hline Department of Respiratory Medicine, National Hospital Organization Iwakuni Clinical Center & Iwakuni, Japan \\
\hline Division of Respiratory Medicine, National Center for Global Health and Medicine & Tokyo, Japan \\
\hline Department of Medical Oncology, Hokkaido University Hospital & Sapporo, Japan \\
\hline Department of Thoracic Oncology, Saitama Cancer Center & Saitama, Japan \\
\hline Department of Respiratory Medicine, Kitasato University Hospital & Sagamihara, Japan \\
\hline Department of Respiratory Medicine, Saitama Cardiovascular and Respiratory Center & Kumagaya, Japan \\
\hline Department of Respiratory Medicine, Otemae Hospital & Osaka, Japan \\
\hline Department of Respiratory Medicine, Kyoto Katsura Hospital & Kyoto, Japan \\
\hline Department of Respiratory Medicine, Tohoku University Hospital & Sendai, Japan \\
\hline
\end{tabular}

Table S2 Post-protocol treatment regimens used in $\geq 5 \%$ of patients

\begin{tabular}{lcc}
\hline Regimen, $\mathrm{n}(\%)$ & CisPemBev, N=131 & CarPacBev, N=66 \\
\hline $2^{\text {nd }}$ line & $101(77)$ & $51(77)$ \\
$3^{\text {rd }}$ line & $60(46)$ & $33(50)$ \\
$4^{\text {th }}$ line & $29(22)$ & $18(27)$ \\
Cisplatin/carboplatin & $16(12)$ & $11(17)$ \\
Docetaxel & $59(45)$ & $23(35)$ \\
Pemetrexed & $10(8)$ & $40(61)$ \\
Paclitaxel/nab-paclitaxel & $21(16)$ & $1(2)$ \\
S-1 & $25(19)$ & $6(9)$ \\
Bevacizumab & $7(5)$ & $7(11)$ \\
Ramucirumab & $27(21)$ & $13(20)$ \\
Nivolumab/pembrolizumab & $64(49)$ & $31(47)$ \\
\hline
\end{tabular}

CarPacBev, carboplatin + paclitaxel + bevacizumab; CisPemBev, cisplatin + pemetrexed + bevacizumab. 